\title{
Density-functional Based Tight-binding Calculations on Thiophene Polymorphism
}

\author{
J. WIDANY*, G. DAMINELLI, A. DI CARLO and P. LUGLI \\ Department of Electronic Engineering, University of Rome "Tor Vergata", \\ Via di Tor Vergata 110, 1-00133 Rome, Italy
}

\begin{abstract}
Total energy calculations based on a density-functional tight-binding scheme have been performed on polymorphic modifications of various thiophene crystals. The investigated structures include sulphanyl-substituted quater-thiophene and methyl-substituted sexithiophene, in the monoclinic and triclinic modifications. Attention has been focused on the intermolecular interaction between the molecular units. Despite the similarities in the backbone geometries, the strength and nature of intermolecular interaction differs largely in the various polymorphs. Sulphur atoms belonging to the thiophene rings are strongly involved in the interaction. Sulphanyl substituents play an important role, while methyl groups do not contribute. The strength of intermolecular interaction is not a direct function of atom distance.
\end{abstract}

Keywords: Organic semiconductors; Oligothiophene; Polymorphic crystals; Intermolecular interaction; Total energy calculations; Density functional theory; Tight-binding

\section{INTRODUCTION}

Conjugated organic oligomers and polymers have attracted much scientific interest for applications in electronic and opto-electronic devices [1-3]. While the properties of oligomers in solution have been investigated in details [4], little is known about electronic properties in the solid state, such as single crystals or crystalline thin films. However, this theoretical insight is a prerequisite for successful tuning of the device properties.

Among organic compounds, thiophene oligomers are considered as promising materials for opto-electronic applications [5-7]. This is due to the simplicity of their preparation and functionalization, their chemical stability and favorable electronic properties. While they are successfully used in field effect transistors, oligothiophenebased light emitting diodes suffer from low quantum efficiency (usually below $2 \%$ ). Efficiency enhancement can be achieved through chemical tailoring, with an appropriate choice of substituents. Recently, the preparation of new materials with photo-luminescence efficiencies up to $37 \%$ has been reported [8].

For two of the recently synthesized materials, polymorphic modifications have been found [911], which are of special interest for theoretical

*Corresponding author. Tel.: +3906 7259 7367, Fax: +3906 2020519, e-mail: wid@eln.uniroma2.it 
investigations. Although the molecular units in the polymorphs are of the same type, the arrangement in the crystal varies. Thus, they give the possibility of studying directly the effects of crystal packing.

In the present work we present the results of total energy calculations on thiophene polymorphs. We describe the different kind of intermolecular interaction in the crystals and discuss the role of substituents.

\section{METHOD}

To determine the total energy and the interatomic forces for a given set of atomic positions we use a non-orthogonal tight-binding (TB) scheme. The method allows for calculations within large super cells (up to 1000 atoms) and the inclusion of 3dimensional periodic boundary conditions. In contrast to most other TB methods, the Hamiltonian and overlap matrix elements are not obtained from a fit to an experimental database, but calculated within self-consistent local density approximation (LDA). A complete description of the method can be found in Refs. [12,13].

The total energy $E_{\text {tot }}$ for a given set of coordinates $\left\{\mathbf{R}_{k}\right\}$ can be approximated as a sum of the band structure energy (which is the sum of the occupied Kohn-Sham eigenvalues) and a sum of short-range repulsive pair-potentials:

$$
E_{\text {tot }}\left(\left\{\mathbf{R}_{k}\right\}\right)=\sum_{i} n_{i} \varepsilon_{i}\left(\left\{\mathbf{R}_{k}\right\}\right)+\sum_{k<l} V_{\text {rep }}\left(\left|\mathbf{R}_{l}-\mathbf{R}_{k}\right|\right)
$$

Appropriate (pseudo)-atoms of each type are constructed by self-consistent solution of a modified atomic Kohn-Sham equation. The overlap and Hamiltonian matrix elements are calculated out of this basis of atomic wave functions. Several contributions to the Hamiltonian matrix can be neglected, in fact, only two-center elements are dealt with. The so generated matrix elements depend only on the interatomic distance. The short-range repulsive potential is derived again from a self-consistent LDA calculation. Once this set of input data (Hamiltonian and overlap matrix, repulsive potential) for the $\mathrm{TB}$ is generated for each interaction pair, it can be used for the determination of equilibrium geometries and total energies of all scale heteronuclear systems.

To handle charge transfers within the model, long-range Coulomb interaction is included through a self-consistent treatment of the charge density [14]. While a minimal basis set is sufficient for the description of the electronic system of carbon and hydrogen, sulphur requires the inclusions of $d$-orbitals.

\section{OLIGOTHIOPHENE POLYMORPHS}

The following crystals have been investigated: ( $\beta$-tetra-methylsulphanyl)- $\alpha$-quaterthiophene (TMS4T) and ( $\beta$-tetramethyl)- $\alpha$-sexithiophene (TM6T). Both compounds exist in the solid state in two crystalline forms: triclinic (TMS4Tr and TM6Tr), and mono-clinic (TMS4Ty and TM6Ty). To compare the effect of substituents, we also include data of the methyl substituted quaterthiophene (TM4T). The crystalline structure of these compounds has been determined by X-ray characterization $[9-11,15]$.

A remarkable feature of all compounds is the close similarity of the backbone geometry, even though the substituent groups are different. All compounds show an exactly coplanar conformation of the inner thiophene rings. The main difference of the polymorphs derives from the tilting of the outer rings. The triclinic compounds consist of more planar configurations than the monoclinic ones. The outer rings in TMS4Tr and the homologue rings in TM6Tr have an anti conformation, the torsion angles with the central units being $153^{\circ}$ and $155^{\circ}$, respectively. In contrast, molecules in the monoclinic crystals are strongly twisted with torsion angles of $56^{\circ}$ and $55^{\circ}$ for TMS4Ty and TM6Ty, respectively, thus leading to a syn conformation. 
The different molecular structure of the compounds leads to completely different crystal packing: the triclinic forms exhibit a stacked supramolecular structure, while the monoclinic ones show a nearly herringbone structure.

An experimental study on the photoluminescence and quantum efficiency of the polymorphs reveals strong differences [16]. TSM4Tr has the highest quantum efficiency (14\%), compared to $1 \%$ for the TSM4Ty. Values are more homogeneous for the sexi-thiophene: $4 \%$ and $2 \%$ for TM6Tr and TM6Ty, respectively.

\section{RESULTS}

The crystal geometry has been optimized applying conjugate gradient techniques. Deviations of the optimized bond lengths from the initial X-ray values are less than $0.02 \AA$ for $\mathrm{C}-\mathrm{C}$ bonds and less than $0.01 \AA$ for $\mathrm{C}-\mathrm{S}$ bonds. The $\mathrm{C}-\mathrm{S}-\mathrm{C}$ bond angles are found slightly enlarged $\left(<1^{\circ}\right)$. The torsion angles between thiophene rings are preserved.

Results of the total energy calculations are given in Table I. The small differences in energy between TMS4Tr/TMS4Ty and TM6Tr/TM6Ty confirm the polymorphic character of the crystals. In both cases, the triclinic modification are more stable than the monoclinic ones, in agreement with data reported in Ref. [11].

We also give the total energy of the single molecule, whose geometry has been extracted from the optimized crystal structure. The geometry obtained with this procedure does not necessarily

TABLE I Comparison of the total energy of crystals and single molecules. Energies in $\mathrm{eV} /$ molecule (containing 46, 42 and 56 atoms, respectively)

\begin{tabular}{lccc}
\hline & $\mathrm{E}_{\text {crystal }}$ & $\mathrm{E}_{\text {single mol }}$ & $\mathrm{E}_{i i}$ \\
\hline TMS4Tr & -1649.25 & -1648.67 & 0.58 \\
TMS4Ty & -1648.98 & -1648.39 & 0.59 \\
TM4T & -1384.03 & -1383.76 & 0.27 \\
TM6Tr & -1932.53 & -1932.02 & 0.51 \\
TM6Ty & -1932.16 & -1931.74 & 0.42 \\
\hline
\end{tabular}

represent an optimized structure, but allows for the determination of the intermolecular interaction. Although the method in general tends to underestimate intermolecular forces, the energy difference $\mathrm{E}_{i i}$ between crystal and single molecule refers directly to the extent of intermolecular interactions.

Despite the fact that TMS4T polymorphs have similar $\mathrm{E}_{i i}$ values, the wave function overlap in the two crystals is completely different. Within the tight-binding approach the overlap can be written as bond order term, which is defined, between two neighboring atoms $A$ and $B$, as:

$$
P_{A B}=\sum_{i} n_{i} \sum_{\mu(A)} \sum_{\nu(B)} c_{\mu}^{i} S_{\mu \nu} c_{\nu}^{i}
$$

with $n_{i}$ being the occupation number of molecular orbital $i, c_{\mu, \nu}^{i}$ the eigenvector-matrix elements $\mu, \nu$ for $i$ and $S_{\mu \nu}$ the overlap matrix element $\mu, \nu$. Compared to bonds within the molecule, the bond order of weak intermolecular coupling is found to be 2 orders of magnitude smaller.

The almost planar stacking of the TMS4Tr results in an extended intermolecular $\pi-\pi$ interaction. This involves sulphur atoms on the thiophene units and conjugated carbon atoms on the neighboring molecules. A similar interaction is prevented by the strong torsion in the TMS4Ty structure. Figure 1 demonstrates this different behavior: One entire molecule within each crystal is shown (as sticks) with all interacting atoms on neighboring molecules (as balls). In the upper part we see the parallel stacking of the TMST4r, the $\pi-\pi$ interaction occurring with the fragment below the main molecule. In TMST4y, shown in the lower part, the number of interacting centers is clearly smaller than in the previous case and consists mainly of $\mathrm{S}-\mathrm{S}$ and $\mathrm{S}-\mathrm{H}$ bridges.

The more extended interaction occurring in TMS4Tr crystals does not result in a higher $\mathrm{E}_{i i}$ value. This can be related to steric effects of the substituents, which introduce a negative energy contribution. In fact, TMS4Tr is the only case where the molecule inside the crystal has not an 


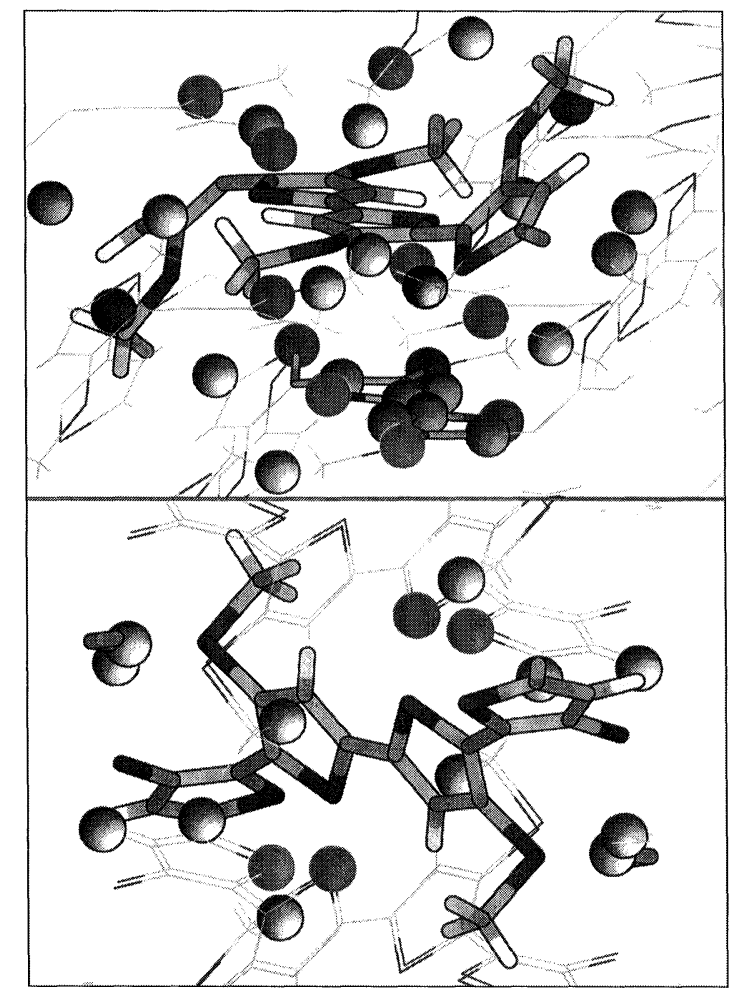

FIGURE 1 Wave function overlap in TMS4Tr (upper part) and TMS4Ty (lower part). Single molecule (depicted as sticks) with interacting neighbor atoms: sulphur (black), carbon (grey) and hydrogen (white).

optimized structure. We therefore expect that the distortion in the single molecule partially cancel out the large energy gain due to intermolecular interaction.

In both TMS4T crystals, substituents contribute greatly to the overall intermolecular interaction. On the other hand, in TM4T no wave function overlap occurs with the methyl substituents. Interaction is determined by a completely different mechanism. The outer rings of neighboring molecules have an exactly planar configuration, which results in $\mathrm{S}-\mathrm{H}$ overlap within an edge-to-edge alignment, as shown in Figure 2. The inner units shows only weak $\mathrm{S}-\mathrm{H}$ interactions.

In TM6Tr, sulphur atoms of the terminating rings interact with the entire inner unit of a neighboring molecule, within a face-to-face alignment (see upper part of Fig. 3). Thus, the outermost

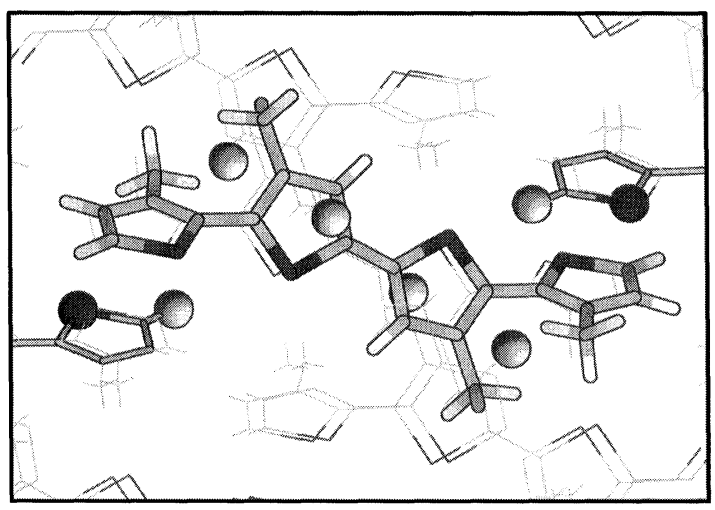

FIGURE 2 Wave function overlap in TM4T. Neighbor rings with interacting atoms are shown as thin sticks.

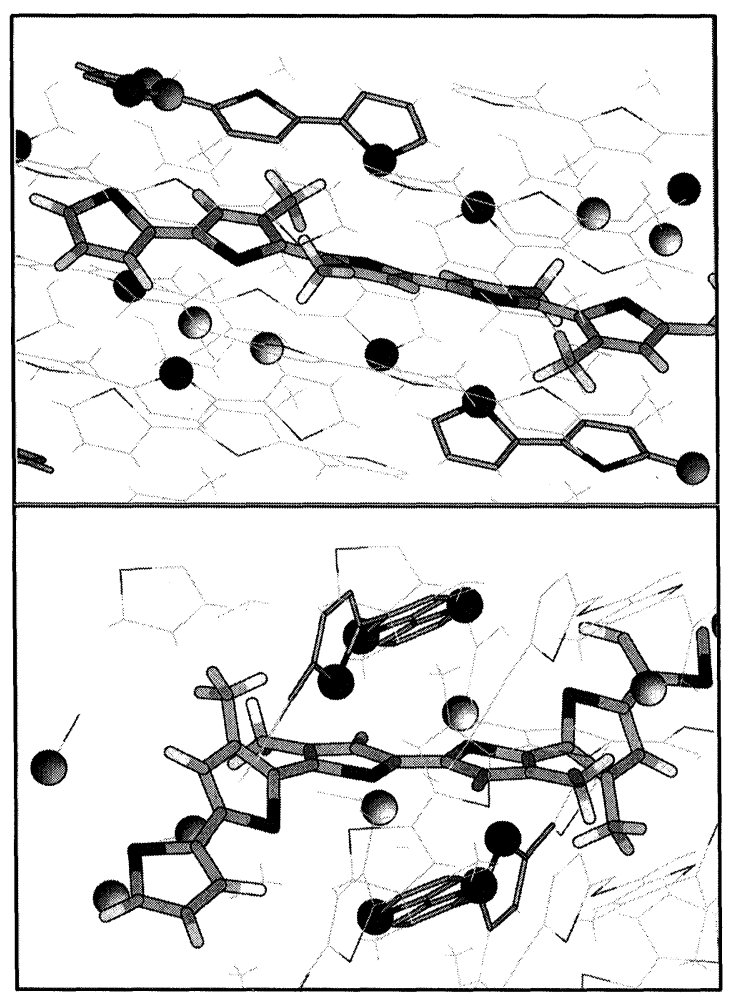

FIGURE 3 Wave function overlap in TM6Tr (upper part) and TM6Ty (lower part).

rings are of crucial importance to the intermolecular interaction. The anti conformation of the six thiophene rings leads to an optimized wave function overlap between sulphur orbitals and the conjugated $\pi$-system. 
The polymorphic modification TM6Ty is characterized by interaction of the sulphur atoms on the inner rings with sulphur atoms on neighboring molecules, as shown in the lower part of Figure 3. The external units of the molecule are less involved in the interaction, leading only to $\mathrm{S}-\mathrm{H}$ intermolecular bridges. In both cases, the methyl groups do not contribute to the intermolecular forces.

The energy gain $\mathrm{E}_{i i}$ through crystallization is higher for triclinic TM6Tr than for monoclinic TM6Ty, in agreement with the larger extension of intermolecular interaction. In contrast to the quater-thiophene, molecular structures in both sexithiophene crystals have an optimized geometry.

Finally, it should be noted that the strength of interactions is not a direct function of the distance of interacting atoms. The orientation and the alignment of interacting centers is of crucial importance.

\section{CONCLUSIONS}

The theoretical investigation shows that a similar backbone structure does not indicate a similar nature of intermolecular interaction. Moreover, a short distance between two atoms is not necessarily an indication for a strong interaction. It is therefore not possible to predict intermolecular interactions only on the basis of geometrical data, i.e., $\mathrm{X}$-ray measurements.

The difference between TM4T and TMS4T shows the importance of the type of substituents. The introduction of sulphanyl groups changes completely the molecular packing. In contrast to the methyl groups, sulphanyl substituents act as strong interaction centers. This can be ascribed to the properties of sulphur atoms, with its electrodonating character (related to the presence of electron lonely pairs) and the possible electron retro-donation (through population of $d$-orbitals).

The structure with the strongest $\pi-\pi$ interaction, TMS4Tr, exhibits the highest quantum efficiency. This result is in contradiction to the common believe that a strong $\pi$ overlap of the molecule suppresses the luminescence and lowers the efficiency. Further studies are needed to establish a clear relation between molecular packing and optical properties of the structures.

\section{Acknowledgements}

We gratefully acknowledge G. Jungnickel, M. Elstner and Th. Frauenheim, University of Paderborn, Germany, for the DYLAX-code and the sulphur Slater-Koster data; G. Gigli, University of Lecce, Italy, and G. Barbarella, CNR Bologna, Italy, for the experimental data on thiophene crystals.

\section{References}

[1] Garnier, F., Hajlaoui, R., Yassar, A. and Srivastata, P. (1994). Science, 264, 1684.

[2] Uchiyama, K., Akimichi, H., Hotta, S., Hoge, N. and Sakaki, H. (1994). Mat. Res. Soc. Symp. Proc., 328, 389.

[3] Baigent, D. R., Friend, R. H., Lee, J. K. and Schrock, R. R. (1995). Synth. Met., 71, 2171.

[4] Birmbaum, D., Fichou, D. and Kohler, B. E. (1992). J. Chem. Phys., 96, 165.

[5] Kanemitsu, Y., Suzuki, K., Matsumoto, Y., Tomiuchi, Y., Shiraishi, Y. and Kuroda, M. (1994). Phys. Rev. B, 50, 2301.

[6] Beljonne, D., Cornil, J., Friend, R. H., Janssen, R. A. J. and Brédas, J. L. (1996). J. Am. Chem. Soc., 118, 6453.

[7] Gigli, G., Rinaldi, R., Lomascolo, M., Cingolani, R., Barbarella, G. and Zambianchi, M. (1998). Appl. Phys. Lett., 72, 1013.

[8] Gigli, G., Barbarella, G., Favaretto, L., Cacialli, F. and Cingolani, R. (1999). Appl. Phys. Lett., 75, 439.

[9] Barbarella, G., Zambianchi, M., Montserrat del Fresnol Marimon, Antolini, L. and Bongini, A. (1997). $A d v$. Mater., 9, 484.

[10] Barbarella, G., Zambianchi, M., Di Toro, R., Colonna, M., Antolini, L. and Bongini, A. (1996). Adv. Mater., 8, 327.

[11] Barbarella, G., Zambianchi, M., Antolini, L., Ostoja, P., Maccagnani, P., Bongini, A., Marseglia, E. A., Tedesco, B., Gigli, G. and Cingolani, R. (1999). J. Am. Chem. Soc., 121, 8920 .

[12] Porezag, D., Frauenheim, Th., Köhler, Th., Seifert, G. and Kaschner, R. (1995). Phys. Rev. B, 51, 12947.

[13] Seifert, G., Porezag, D. and Frauenheim, Th. (1996). Int. J. Quantum Chem., 98, 85.

[14] Elstner, M., Porezag, D., Jungnickel, G., Elsner, J., Haugk, M., Frauenheim, Th., Suhai, S. and Seifert, G. (1998). Phys. Rev. B, 58, 7260.

[15] Barbarella, G., Zambianchi, M., Bongini, A. and Antolini, L. (1993). Adv. Mater., 5, 834.

[16] Gigli, G., Phys. Rev. Lett., in press. 

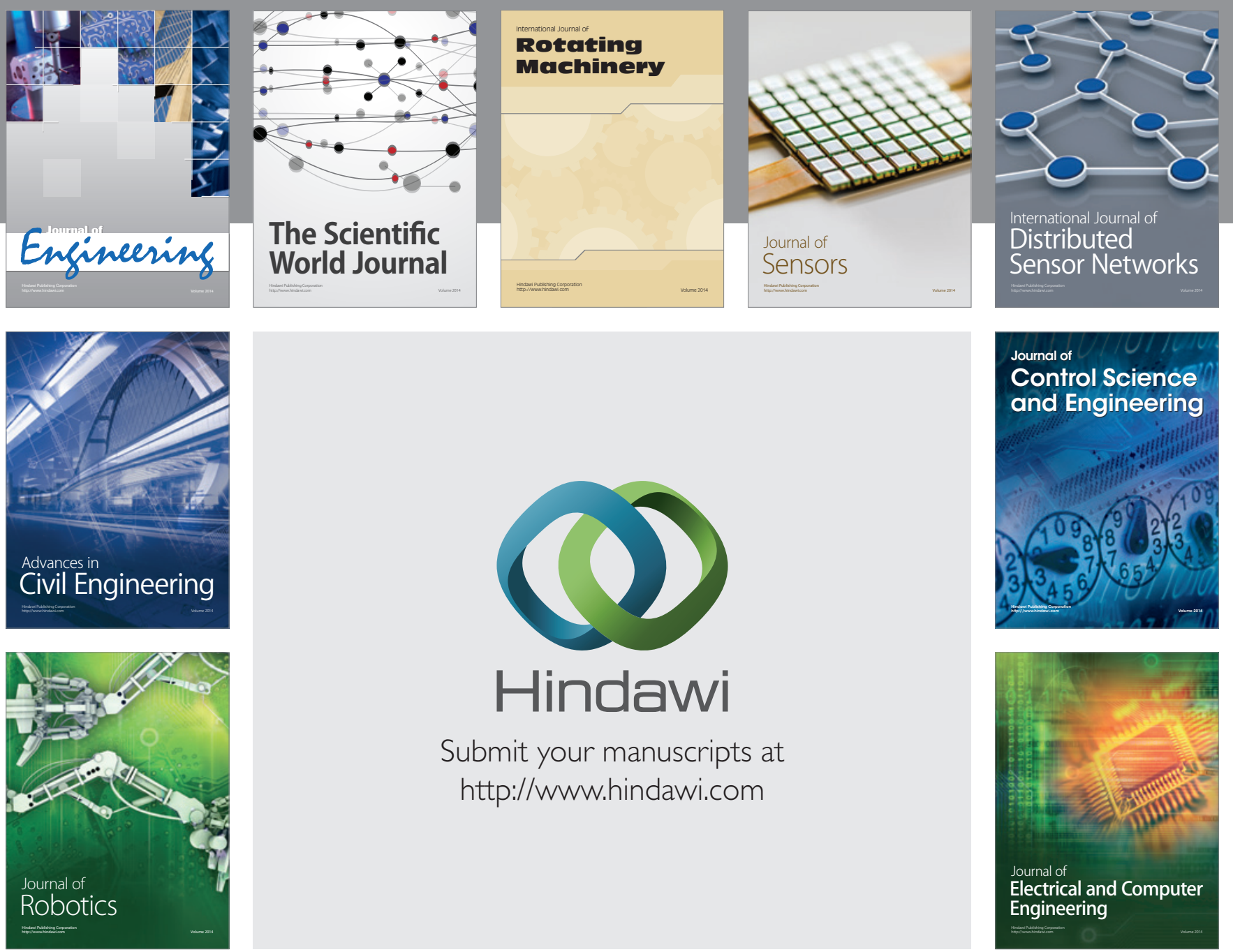

Submit your manuscripts at

http://www.hindawi.com
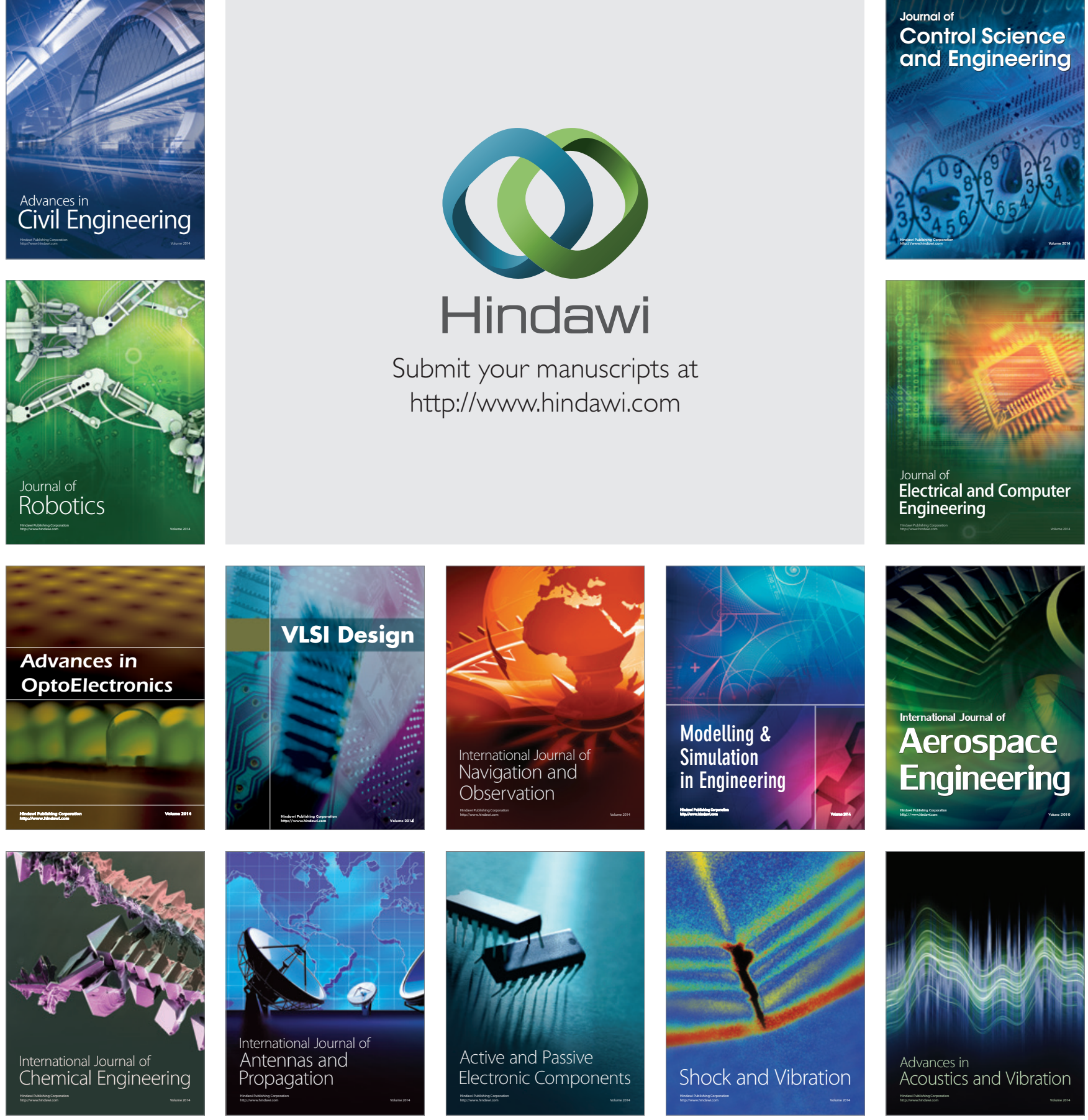